\title{
Design of GNSS Performance Analysis and Simulation Tools as a Web Portal
}

\author{
Srdjan Tadic, Gregory Moura, and Thierry Trichaud
}

\begin{abstract}
This paper considers design of a web-portal for the validation of behavior of GNSS applications in different environments. The tool provides the positioning performance analysis and a comparison to benchmark devices. Web-portal incorporates a 3D synthetic data generator to compute the propagation and the reception of radio-navigation signals in a 3D virtual environment. This radio propagation simulator uses ray-tracing to calculate interactions between the GNSS signal and the local environment. For faster execution on a GPU platform, the simulator uses BVH optimization. The work is verified in field trials and by using reference software.
\end{abstract}

Keywords - GNSS, EGNOS, datapool, simulation, BVH.

\section{INTRODUCTION}

$\mathrm{B}$ EFORE deployment and the commercialization of every new positioning product/application, manufacturers or developers need to perform long and often expensive field trials in targeted operational environments in order to assess and certify future behavior. The community trust on GNSS (Global Navigation Satellite System) is so high that more and more applications based on satellite navigation are now safety critical. With the increase of criticality level of GNSS applications, product developers are requesting a relevant mean to predict the performances and reliability of future applications. For example, one will be interested in knowing the GNSS performances in the city of Berlin for its critical application for guiding blind people while another one would like to predict the accuracy of its road toll collection system over Serbia. In that context, a system called the "European Geostationary Navigation Overlay Service" (EGNOS) is introduced to supplement the GNSS systems by reporting on the reliability and accuracy of the signals primarily in aeronautics, along with providing differential corrections to GNSS.

This work will present design considerations of a data

Paper received March 13, 2014; revised June 1, 2014; accepted June 18, 2014. Date of publication November 15, 2014. The associate editor coordinating the review of this manuscript and approving it for publication was Prof. Aleksandar Nešković.

This paper is a revised and expanded version of the paper presented at the 21th Telecommunications Forum TELFOR 2013.

The research leading to these results is co-funded by the European Union's Seventh Framework Programme (grant agreement no 296379).

Srdjan Tadic, School of Electrical Engineering University of Belgrade, Serbia (e-mail: srdjan.tadic@gmail.com).

Gregory Moura, Oktal Synthetic Environment, Toulouse, France (e-mail: gregory.moura@oktal-se.fr).

Thierry Trichaud, M3-Systems, Toulouse, France, (e-mail: trichaud@m3systems.net). pool of worldwide GNSS measurements that should enable low cost tests campaigns, performances predictions and simulations of new applications and adaptation of the EGNOS integrity concept and inspiring its use in new application domains. It also aims at ensuring the reliability of retrieval and use of digital resources across applications and platforms and at scaling up GNSS data analysis to keep pace with the extremely large data volumes of tests campaigns.

\section{PRIOR ART}

A partial list of existing GNSS performance analysis solutions is shown in Table 1. Most of the available tools are fully dedicated to the aeronautic domain despite the identified need [1] for use in urban environment. Table 2 summarizes the main GNSS simulation solutions available.

TABLE 1: EXISTING GNSS PERFORMANCE ANALYSIS TOOLS.

\begin{tabular}{|c|c|c|}
\hline Tool & Data & Main services \\
\hline $\begin{array}{l}\text { Magic GNSS } \\
\text { suite [2] }\end{array}$ & $\begin{array}{l}\text { GPS,SBAS } \\
\text { GLONASS } \\
\text { EGNOS }\end{array}$ & $\begin{array}{l}\text { - Orbit determination and time } \\
\text { synchronization } \\
\text { - Ionospheric effects }\end{array}$ \\
\hline GISMO [3] & GPS & $\begin{array}{l}\text { - Availability, accuracy, integrity } \\
\text { - Signal reception/quality stats. } \\
\text { - Identification of faults/failures }\end{array}$ \\
\hline $\begin{array}{l}\text { EUROMON } \\
{[4]}\end{array}$ & $\begin{array}{l}\text { GPS } \\
\text { EGNOS }\end{array}$ & $\begin{array}{l}\text { - Quality, availability } \\
\text { - Corrections tested on } \\
\text { continuity, integrity, acc., avail. }\end{array}$ \\
\hline $\begin{array}{l}\text { GNSS } \\
\text { GLOBAL } \\
\text { SIMULATOR } \\
{[5]}\end{array}$ & GPS & $\begin{array}{l}\text { - Developing and testing GNSS } \\
\text { algorithms performance } \\
\text { - Reliability, precision, accuracy } \\
\text { - Signal proc. intermediate result }\end{array}$ \\
\hline $\begin{array}{l}\text { GNSS Perf. } \\
\text { Moni. Sys. [6] }\end{array}$ & GPS & $\begin{array}{l}\text { - Accuracy, integrity, availability } \\
\text { - Real-time monitoring }\end{array}$ \\
\hline
\end{tabular}

TABLE 2: EXISTING GNSS PROPAGATION SIMULATORS

\begin{tabular}{|c|c|c|}
\hline Tool & Type & Main services \\
\hline $\begin{array}{l}\text { WinProp } \\
{[7]}\end{array}$ & $\begin{array}{l}\text { Empirical } \\
\text { deterministic }\end{array}$ & $\begin{array}{l}\text { Propagation models: } \\
\text { - Empirical Two-Ray, } \\
\text { - Deterministic Two Ray, } \\
\text { - Okumura-Hata, } \\
\text { - Walfisch-Ikegami }\end{array}$ \\
\hline $\begin{array}{l}\text { NavTK } \\
{[8]}\end{array}$ & Statistical & $\begin{array}{l}\text { - Direct visibilities + multipaths } \\
\text { - Compliant with STK formats } \\
\text { - 2D coverages and maps }\end{array}$ \\
\hline $\begin{array}{l}\text { Polaris } \\
\text { [9] }\end{array}$ & Statistical & $\begin{array}{l}\text { - GPS, GALILEO, SBAS. } \\
\text { - Models hybridized receivers } \\
\text { - Direct visibilities only }\end{array}$ \\
\hline
\end{tabular}

"WinProp" is a RF network planning software that 
computes propagation losses considering the channel characteristics. Emitters can be terrestrial beacons or satellites. Separate modules are managing propagations in different types of environment (e.g. rural, urban areas, indoor, etc.). Simulation provides received power, delay, dilution of precision (DOP), phase, 2D reception maps etc.

The "Navigation Tool Kit" (NavTK) is focused on the Global Positioning System (GPS) study. Software models the different versions of the GPS satellites (Block II, II, IIA, IIR, IIR-M, and IIF) to predict the availability of the system and its level of accuracy and confidence.

Satellites' payloads are also modeled and the user can trigger hypothetical satellite errors and outages to inspect impacts on the reception of the signal. NavTk computes direct visibility of the satellites and has a statistical algorithm to model the interactions between the signal and the receiver environment.

Simulation tool "Polaris" estimates the accuracy, the availability, DOP, the Number of visible satellites (NSV) and the Protection Level (PL) for a target system, the user's location and its environment. The tool allows the generation and management of 3D-buildings and computes shadowing effects on the GNSS coverage, Satellite and Ground-Based Augmentation Systems (SBAS, GBAS) or some other local system.

\section{PROPOSED SOLUTION}

GAPFILLER [10] is web-based software that includes GNSS trajectories and the associated data from all available areas. The portal users can access and retrieve online content and services through open Application Programming Interface (API) based on NMEA (National Marine Electronics Association) and RINEX (Receiver Independent Exchange Format). Optionally, the outputs of inertial sensors can be associated with the recorded GNSS data. This enables the evaluation of the coupling algorithms of Inertial Navigation Systems (INS) and GNSS in specific areas with various sensors, antennas and receivers.

The main functionalities of data portal (Fig. 1) are:

- An access to the data gathered by different receivers.

- A receiver emulation module, which, by using the GNSS data from the data pool, emulates the behavior/ integrity of the application on different trajectories.

- A framework of performance analysis tools allowing statistic comparison of GNSS performances.

- A 3D synthetic data generator enabling to compute the propagation and the reception of radio-navigation signals in a $3 \mathrm{D}$ virtual environment.

\section{Performance Analysis module}

The performance analysis module is hosted on the web-server for the human-machine interface part, and on a separate dedicated back-end server for processing and load balancing. It contains:

- statictics component,

- hybridization component - enhancement by coupling with an inertial measurement unit (IMU) or using Differential GPS or Precise Point Positioning (PPP),

- cross-comparison component.

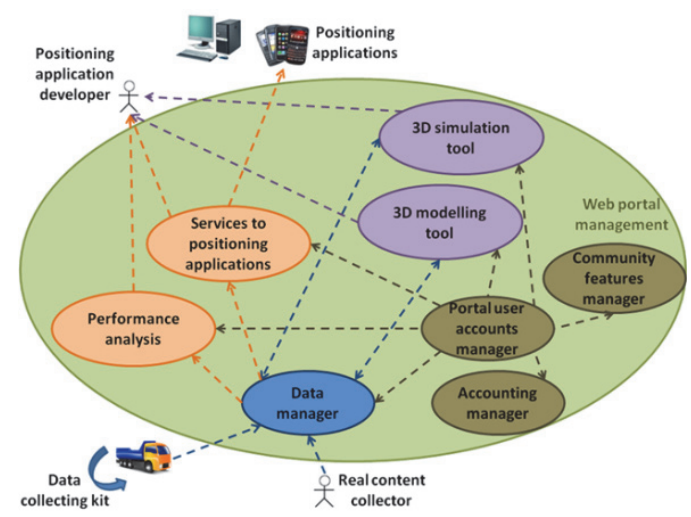

Fig. 1 GAPFILLER data portal functional overview.

The statistics service uses stored RINEX or NMEA log files to produce KML or GPX files that cover selected statistic parameters (DOP, Quality of Service, etc.). An example of statistics module output is shown in Fig. 2.

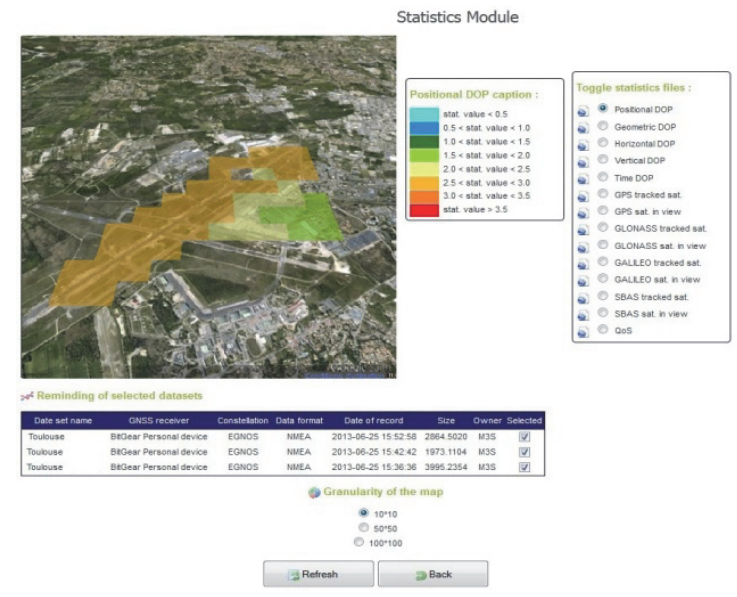

Fig. 2 Statistics module web-report.

A cross comparison feature enables users to further benchmark their devices using reference trajectories recorded using high performance equipment and/or with the use of post processing corrections (Fig. 3). It is able to compute the integrity level of device positioning, the along-track, cross-track error statistics and distribution.

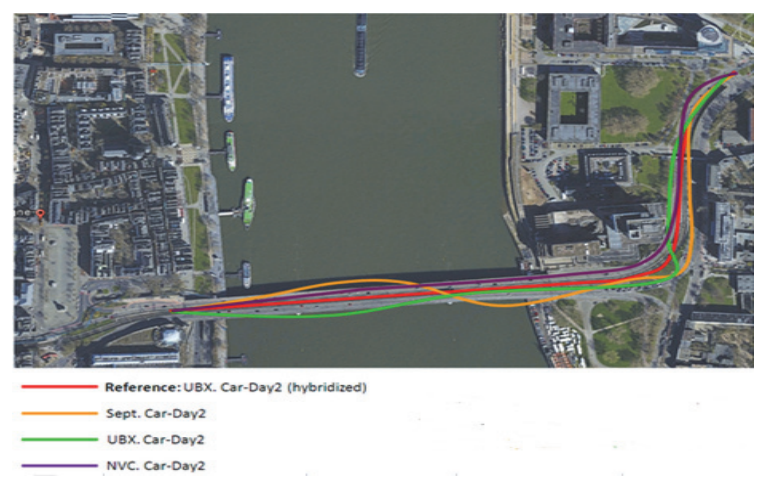

Fig. 3 Cross-comparison tool - an example.

Integrity is the measure of the trust that can be placed in the correctness of the information supplied by a navigation system. The integrity includes the ability of the system to provide timely warnings to users when the system should not be used for navigation. The integrity risk is the probability that, at any moment, the position 
error exceeds the "protection level" (PL). This probability is defined by the user and is taken into account during the protection level computation.

Fig. 4 illustrates different configurations where the system is (not) available and/or (not) safe considering the true position error, the estimated protection level, the probability of missed detection (PMD) and the alert limit (AL). The AL value is a user parameter defined to restrict the use of the receiver to a limited range of precision. When the protection level is greater than the user defined alert limit, the system is simply considered unavailable.

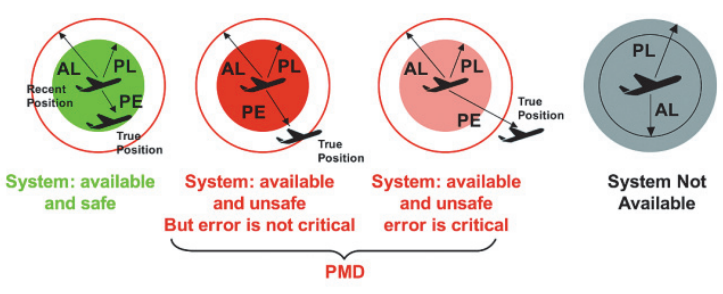

Fig. 4 Integrity mechanism.

The presented cross-comparison feature embeds the NavProc library [11] [12], which is able to handle EGNOS messages and to determine a HPL (Horizontal Protection Level) and a VPL (Vertical Protection Level) for a given position. HPL is the horizontal protection radius at a given PMD probability. It is obtained by combining the standard deviation of the GNSS pseudoranges (estimated according to the receiver measurements and using EGNOS messages) with a coefficient $\mathrm{K}$ which depends on the PMD value: $\mathrm{HPL}=\sigma_{\mathrm{Pos}} \mathrm{K}(\mathrm{PMD})$. The user is able to observe (Fig. 5):

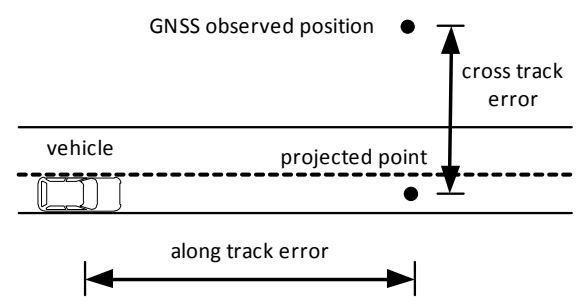

Fig. 5 Across track and along track errors.

- The Horizontal Position Error (HPE) as a measure of the error in a GPS position in the horizontal plane.

- The Along-Track Error as the projection of the HPE on the $2 \mathrm{D}$ axis along which the GNSS receiver is moving.

- The Cross-Track Error as the projection of the HPE on the axis which is perpendicular to the $2 \mathrm{D}$ axis along which the GNSS receiver is moving.

The cross comparison feature provides a mean value, standard deviation, a set of indicative percentiles $(68,95$, 98 and 99) and extremes for all of the listed horizontal position errors. The user is also able to observe:

- cumulative distribution, probability density and the normal distribution of all of the errors (Fig. 6);

- HPE/HPL ratio, which constitutes the integrity risk (Fig. 7).

\section{3D Modeling ANd Simulation ToOL}

Simulation server uses two components to compute the propagation of the GNSS signal in a given area:
- a 3D modeler enabling users to build the local synthetic environment and to assign electromagnetic parameters (permittivity, conductivity, walls thicknesses) to the different materials of the model. A software SEAGETIM-LIGHT is used. It enables integration of various geographical data sources and multisensor fusion. The portal provides users with a library of $3 \mathrm{D}$ mock-ups. Modeler also provides converters to load external 3D scenes.

- a 3D simulation tool computing the propagation of the GNSS signal in the local environment [13] (Fig. 8-10).

The propagation of GNSS signal in a harsh environment is disrupted by the presence of buildings or objects surrounding the receiver. Signal occlusion by various objects is impacting the availability of the navigation system. The performance further degrades because the incoming signal is interacting with the local environment that generates multipaths (reflections, diffractions or transmissions).

Simulations enable detecting harsh areas. A deterministic simulator SE-NAV [14] is integrated in the GAPFILLER framework and is used to populate database with data complementary to those recorded during measurement campaigns. The architecture of the simulation server is described in Fig 11.

The SE-NAV simulates the propagation of GNSS signals in all environments. This software embeds a Raytracing kernel to compute the masks and the multipaths.

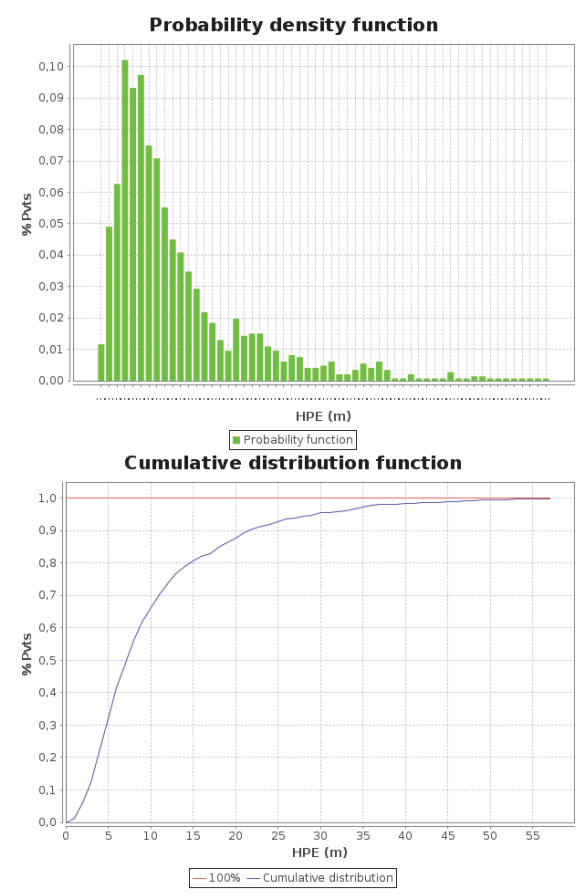

Fig. 6 Cross-comparison module outputs.

1) Raytracing and optimization

A Raytracing algorithm [15] finds intersections between a ray and a predefined geometry. In SE-NAV, obstacle geometries are defined using sets of triangular surfaces (Fig. 12a). For high frequency asymptotic EM simulation, a large number of such intersections has to be computed. A naïve approach would require $\mathrm{N}^{*} \mathrm{M}$ ray-triangle 
intersections - $\mathrm{N}$ being the number of triangles and $\mathrm{M}$ the number of rays. Excluding simple situations, this cost is prohibitive, hence the need for acceleration methods. The Bounding Volume Hierarchy (BVH) [16], [17], an objectbased subdivision structure is chosen. The principle is to build a tree-structure of a set of triangles (Fig. 12b). Each triangle is stored in a leaf of the tree. Each internal node stores for each of its children the bounding volume of the descendant triangles. A BVH is built according to the location of each triangle of the database, trying to minimize the extent and the number of nodes. Once the structure is built, the intersection between a ray and the scene is done by traversing the tree recursively from the root. A child is processed only if the ray intersects its bounding volume. If the two children are traversed, the process is repeated first on the children closest to the ray origin. If the child is a leaf, the ray is intersected with all triangles contained in the leaf, potentially updating the intersection data. Traversal is stopped as soon as no intersection closer than the current one can be found. This method drastically reduces the ray-scene intersection computation time, computing only $\mathrm{O}(\log (N) * M)$ intersections instead of $N^{*} M$ for $N$ triangles and $\mathrm{M}$ rays.
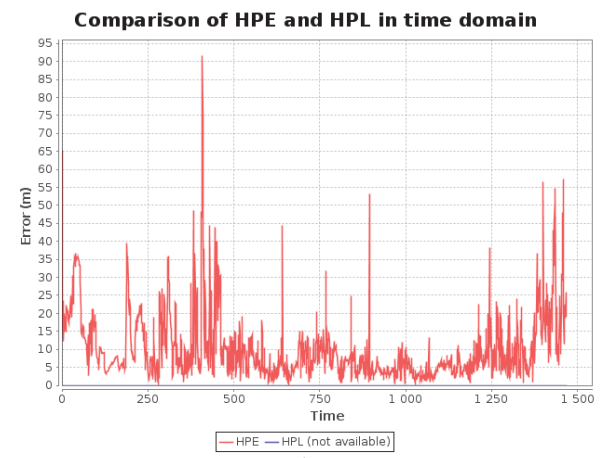

a)

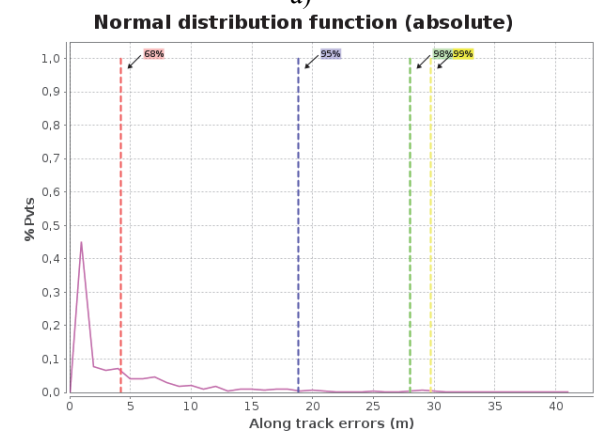

$--68 \%--95 \%--98 \%-99 \%$
b)

Normal distribution function (absolute)

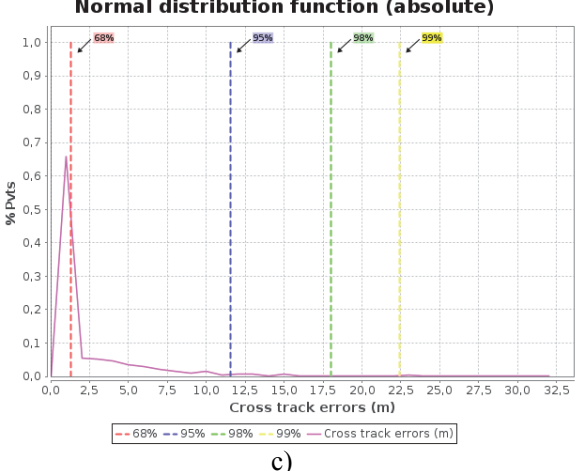

c)

Fig. 7 An example of a) horizontal position error and b-c) distribution of its projections.

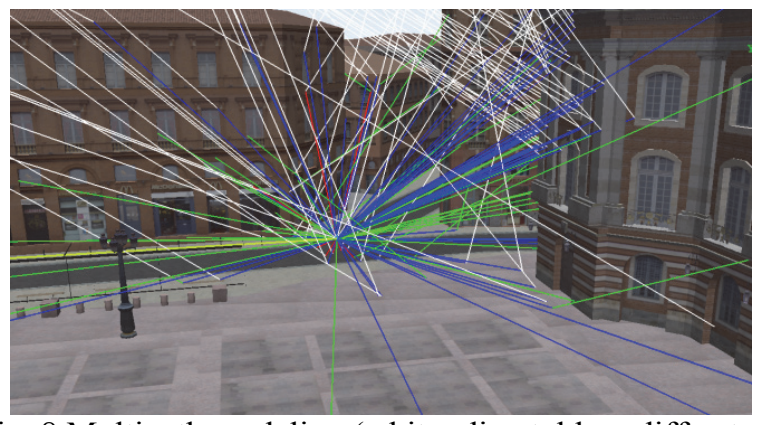

Fig. 8 Multipath modeling (white=direct, blue=diffracted, red $=$ reflected, green $=$ transmitted rays). Red arrows $=$ receiver location.

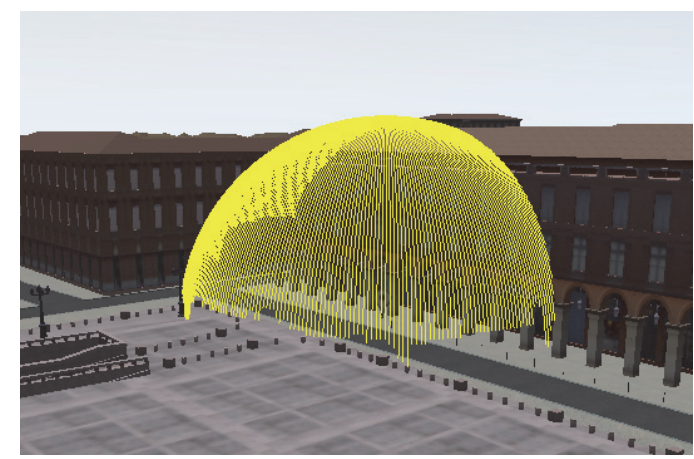

Fig. 9 3D visibility diagram.

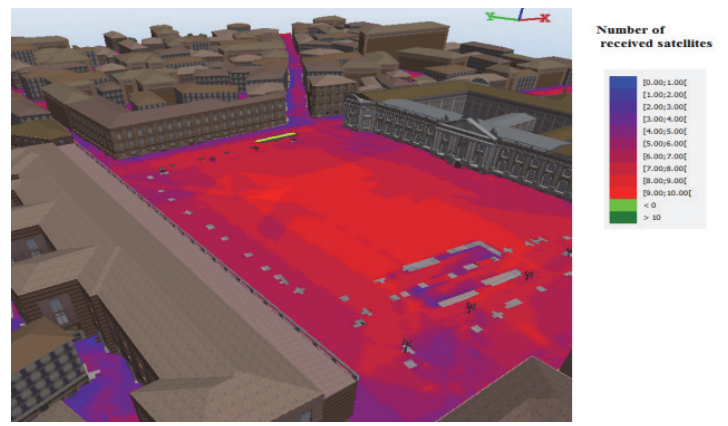

Fig. 10 Example of GPS map - the number of satellites tracked (blue $=$ less than 4 satellites).

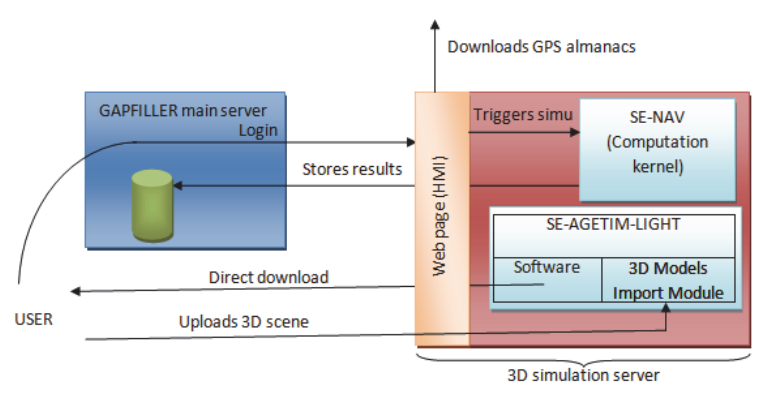

Fig. 11 Simulation server architecture.

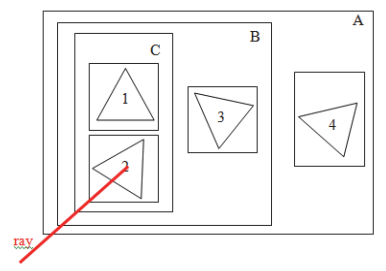

a)

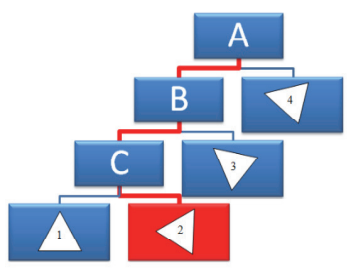

b)
Fig. 12 Bounding-volumes hierarchy. 
Code is optimized for the use of Graphics Processing Unit (GPU) resources. In harsh conditions with the same acceleration method, a GPU-optimized code can be 1000 times faster than a pure-CPU code (Fig. 13).

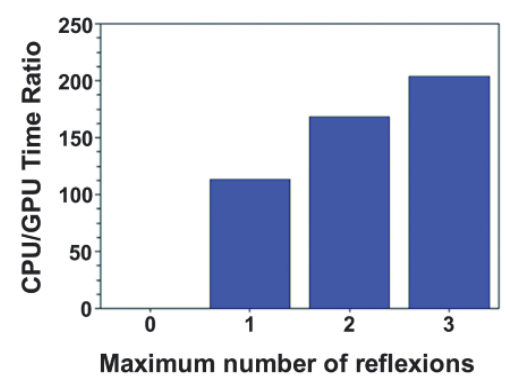

a)

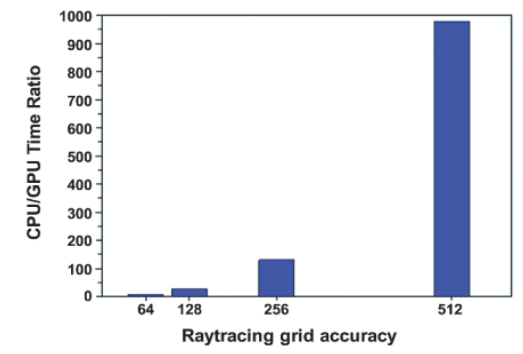

b)

Fig. 13 Computation time GPU vs. CPU depending on a) number of reflections and b) target accuracy.

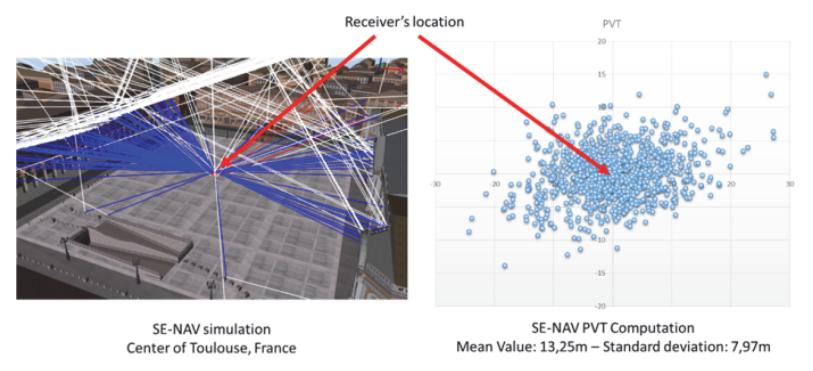

Fig. 14 PVT computation - an example.

\section{2) Physics Modeling}

Geometric optics (GO) have been developed for the analysis of light wave propagation which corresponds to high frequencies. In propagation problems, the GO is valid for frequencies higher than $100 \mathrm{MHz}$ [18]. The simulator uses Geometrical Optics (GO) to model reflections and transmissions and Uniform Theory of Diffraction (UTD) [19] to detect signal's diffractions on the edges of the objects. Simulator uses a deterministic method to calculate and display the geometry of each ray reaching the receiver and then computes the total link budget and errors needed to forecast the local pseudorange [20]. Based on DOP information provided on output and the visibilities of the constellation (line-of-sight, non line-of-sight and hidden satellite), the user can assess the availability of the navigation system and its performance within a given area.

The simulator assesses the main source of attenuations during the signal propagation and computes link budget to provide the received power per multipath and per channel.

\section{3) Link and Error Budgets}

As deterministic software, the simulator provides the power of the received multipath and the global power of a given channel. For computation, the simulator takes into account the following interaction:

- Satellites' cabling losses

- Satellites antenna pattern. The user can choose a mathematical model or load a gain pattern file.

- Free space losses according to the distance and the carrier's frequency.

- Atmospheric losses: Both ionosphere and troposphere are modelled.

- Multipath losses: GO and UTD laws are used to compute the reflection, transmission and diffraction losses of the received multipath.

- Receiver's antenna pattern.

- Receiver's cabling losses.

The simulator models the complete field (modulus and phase) and adds the contributions of the received multipath to compute the global power of a given channel. Fading effect and dynamics of the signal are modelled.

The simulator also computes the PVT (Position, Velocity, Time, Fig. 14) considering the following errors:

- Clock errors,

- Ionosphere delay (Klobuchar and RTCA model)

- Troposphere errors,

- Multipath errors,

- Receiver's errors.

\section{4) Automatic Trajectories Algorithm}

In order to compute the large amount of simulated data, the simulator offers an automatic trajectory service. The objective is to detect roads from the $3 \mathrm{D}$ model and to automatically generate artificial trajectories. Both geometric and physical properties are used to extract roads from a given synthetic environment. This service is an extension of a simulation tool that covers a whole area and provides a user-friendly way to populate the simulated database (Fig. 15).

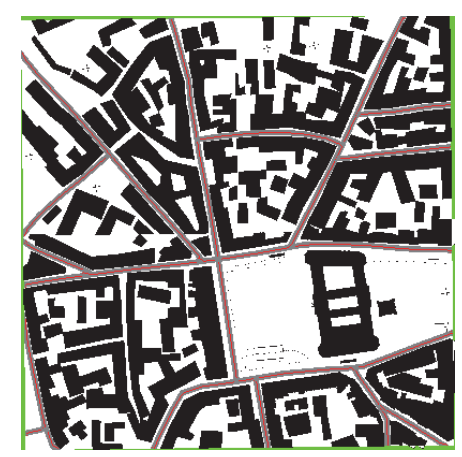

Fig. 15 Top view of a scene: buildings (in black), roads (in grey) and the computed trajectory (in red).

\section{5) Validation}

The validation of the software has been carried out by:

- measurement campaigns in collaboration with Institut Supérieur de l'Aéronautique et de l'Espace, France [21]. - Comparing with referent software SE-RAY-EM [14]. SE-RAY-EM is a fast high frequency analysis method of 
complex 3D targets and environment achieved by coupling EM asymptotic formulations and Shooting and Bouncing Ray approach [22]. An incoming field on a point is computed using both GO and PO (Physical Optics). The tool is awarded with the 2006 Prix Science et Défense in France.

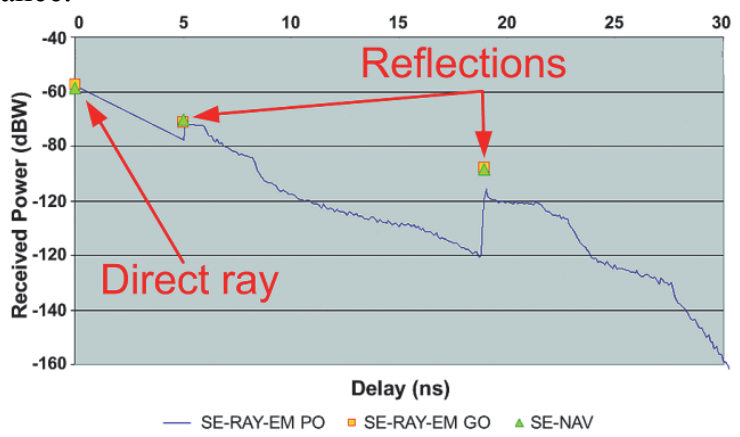

a)

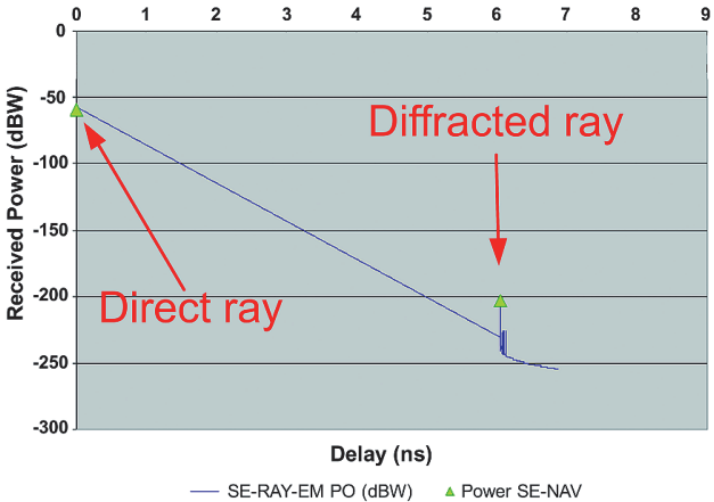

b)

Fig. 16 Comparison of built-in radio propagation simulator and referent tools: power-delay distribution of a) reflections b) diffractions.

Multiple reflections and diffractions were tested on simple dielectric objects (two planes on different configurations). Power-Delay distributions have been built using (Fig. 16):

- Simulator output

- Referent software output without PO contribution

- Referent software output with PO contribution.

The tests have shown a good consistency between two models. Considering GO models, curves show identical multipath delays with the power gap below $0.5 \mathrm{dBW}$. Delay computed with the simulator using GO models matches a referent PO model.

\section{Simulation Process}

The simulator kernel is controlled by a web service in charge of the simulation preparation, triggering and data storage in the database. The process consists of (Fig. 17):

1. A PHP Preprocessing wrapper which builds a temporary scenario file - a simulation description.

2. Simulation - Results (ASCII files in SCILAB format).

3. The PHP PostProcessing wrapper which parses, converts and stores the produced files.

The simulation server provides users with complementary data enabling them to increase the number of reception points and to generate statistical data.

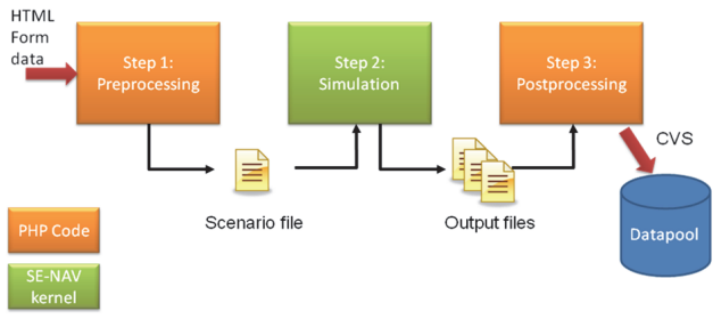

Fig. 17 Simulation process.

\section{CONCLUSION}

The toolset for GNSS performance analysis and simulation in the form of a web-portal offers scalability in handling large data sets gathered in measurement campaigns in any area and optionally shared among users for the easier validation of new positioning-related products and applications. The portal provides an open API based on the NMEA and RINEX messages to increase the usability of data originating from different GNSS technologies. Users can access database data or the data simulated in virtual environment to cross-compare their products and predict future performance.

\section{REFERENCES}

[1] S. Pullen, T. Walter and P. Enge "Integrity for Non-Aviation Users," GPS World, pp. 28-36, July 2011

[2] GMV, Magic GNSS suite. Available:www.gmv.com

[3] NSL, GISMO. Available:www.nsl.eu.com

[4] ALBERDING, EUROMON. Available: www.alberding.eu

[5] SILICOM, Available: www.silicom.biz

[6] DW International, GNSS Perf. Mon. Syst. Avail.: www.dwint.com

[7] AWECommunication, Available: www.awe-communications.com

[8] NavTK/STK. Available: www.agi.com

[9] GMV, Polaris tool. Available: www.gmv.com

[10] GAPFILLER. Available: www.gnss-gapfiller.eu

[11] M3-Systems, www.m3systems.net

[12] J. Soubielle, D. Bétaille, W. Vigneau, (2008) "EGNOS and MEMS sensors tight hybridization for automatic transport systems", Int. Congress and Exh. of Navigation Systems, Technologies and Services - NAVITECH, Moscow, Russia, 2008.

[13] A. Perpey, F. Güell, A. Roy, W. Vigneau, J. Soubielle, J.-P. Mechin, J. Bouffier, T. Chapuis, J. Beas-Garcia, "Precise and Dynamic Road Mapping Creation", $18^{\text {th }}$ World Congress on Intelligent Transportation Systems, Orlando, USA, 2011

[14] Oktal Synthetic Environment, www.oktal-se.fr

[15] A. Bourdeau, M. Sahmoudi, J. Y. Tourneret, "Prediction of NLOS GNSS Signals with a 3D Model of the User Urban Environment", The European Navigation conf., Vienna, Austria, 2013.

[16] Klosowski, James T., Held, Martin, Mitchell, Joseph S. B., Sowizral, Henry and Zikan, Karel. "Efficient Collision Detection Using Bounding Volume Hierarchies of k-DOPs." IEEE Trans. Vis. Comput. Graph. 4 , no. 1 (1998): 21-36.

[17] H.J. Haverkort, "Results on Geometric Networks and Data Structures", Ph.D. thesis, Utrech University, 2004.

[18] P. Pagani, F.T. Talom, P. Pajusco, B. Uguen, Ultra Wide Band Radio Propagation Channel: A Practical Approach, pp. 189-208, John Wiley \& Sons, 2008.

[19] R. G. Kouyoumjian and P. H. Pathak, "A uniform geometrical theory of diffraction for an edge in a perfectly conducting surface," Proc. IEEE, vol. 62, pp. 1448-1461, November 1974.

[20] N. Viandier, D.F. Nahimana, J. Marais, E. Duflos, "GNSS Performance Enhancement in Urban Environment Based on Pseudo-range Error Model", PLANS, Monterey, CA, USA, 2008

[21] Khairol A. B. A., "Reliable GNSS Positioning in Mixed LOS/NLOS Environments Using a 3D Model", The European Navigation conf. ENC'13, Vienna, Austria, 2013.

[22] Y.B. Tao, H. Lin, H.J. Bao, "GPU-Based Shooting and Bouncing Ray Method for Fast RCS Prediction," Antennas and Propagation, IEEE Transactions on, vol.58, no.2, pp.494,502, Feb. 2010. 